\title{
Fields of definition of uniform dessins on quasiplatonic surfaces
}

\author{
Ernesto Girondo, David Torres-Teigell, Jürgen Wolfart ${ }^{1}$
}

Addresses of the authors: Departamento de Matemáticas, Universidad Autónoma de Madrid, Cantoblanco Universidad, Madrid 28049, Spain (E.G., D.T.-T.)

and Instituto de Ciencias Matemáticas, ICMAT-CSIC-UAM-UCM-UC3M (E.G.), Math. Seminar der Goethe Universität, Postfach 111932, D-60054 Frankfurt a. M., Germany (J.W.).

email: ernesto.girondo@uam.es_david.torres@uam.es_wolfart@math.uni-frankfurt.de

Keywords: Compact Riemann surfaces, dessins d'enfants, fields of moduli, fields of definition, Shimura curves EMS classification (2010): Primary 11G32, Secondary 14G35, 14H57, 30F10, 30F35

Abstract. In previous work the authors introduced certain Shimura curves that possess different uniform dessins d'enfants (equivalently, uniform Belyi functions). They are all quasiplatonic and therefore they can be defined over its field of moduli. In this paper the authors determine fields of moduli and some fields of definition of these curves and their related uniform dessins.

This is an extended version of the paper with the same title to appear in Contemporary Mathematics, extended by an appendix containing some more information about the field of definition of Shimura congruence curves of prime power level.

\section{Introduction}

Dessins can be considered as hypermaps on compact oriented two-manifolds, that is bipartite graphs cutting the underlying surface in finitely many simply connected cells. In the special case that all white (or all black) vertices have valency 2 , we may omit these vertices and therefore we are in the classical group theoretic and geometric-topological theory of maps which can be traced back to the classification of platonic solids.

More recently, these objects attracted wider interest by two observations of Grothendieck [12] who invented the term dessins d'enfants for them.

1. Dessins arise in a very natural way on all smooth complex projective algebraic curves defined over number fields: by a result of Belyi [1], there exist non-constant meromorphic functions

$$
\beta: C \rightarrow \mathbb{P}^{1}(\mathbb{C})
$$

\footnotetext{
${ }^{1}$ The first two authors were partially supported by a MICINN grant MTM2012-31973. The second author was partially supported by the Alexander von Humboldt Foundation. The last author was supported by DFG project Wo 199/4-1
} 
on a curve $C$ (equivalently, a compact Riemann surface) ramified at most over $0,1, \infty$ precisely if $C$ can be defined over a number field. Such functions are called Belyi functions and, consequently, algebraic curves defined over $\overline{\mathbb{Q}}$ are also called Belyi curves. In this case, the $\beta$-preimage of the real interval $[0,1]$ defines a bipartite graph on $C$ which is a dessin.

2. Conversely, if we start with an arbitrary topological hypermap on an oriented compact two-manifold, there is a unique conformal structure on the manifold, corresponding to an algebraic curve defined over a number field, together with a Belyi function corresponding to this dessin. A first construction of this conformal structure - at least in the map case - was given by Singerman [18] even before the term dessin was coined.

3. A third important aspect gives a link to the uniformisation of Riemann surfaces, see for example [23]: Belyi functions on a curve $C$ correspond to Fuchsian group inclusions $\Gamma<\Delta(r, s, t)$, where $\Gamma$ is a finite index subgroup of a Fuchsian triangle group $\Delta=\Delta(r, s, t)$ such that $C$ can be written as the quotient $\Gamma \backslash \mathbb{H}$, where $\mathbb{H}$ denotes the upper half plane. With a suitable identification of the target quotient space with the Riemann sphere (the fixed points of $\Delta$ have to be identified with $0,1, \infty)$, the Belyi function is then the canonical quotient mapping

$$
\begin{aligned}
\beta: \Gamma \backslash \mathbb{H} & \rightarrow \Delta \backslash \mathbb{H} \\
\Gamma z & \mapsto \Delta z
\end{aligned}
$$

Dessins should therefore encode all relevant properties of the underlying Riemann surfaces, in particular their defining equations as algebraic curves and the explicit form of the Belyi function of the dessin. Finding these data from the combinatorial structure of the dessin is however a very hard problem, so one is already satisfied to determine the field of moduli and the minimal field of definition for $C$ and the Belyi pair $(C, \beta)$, if such minimal field exists; for precise definitions see the next section. The present article focuses on these objects in the case of certain Shimura curves containing different uniform dessins. Why are these dessins so interesting?

Although dessins define their underlying curves uniquely, on a Belyi curve one can always construct an infinity of Belyi functions, hence an infinity of dessins. How are these different dessins linked to each other? Can one get uniqueness of dessins by imposing additional conditions?

One can in fact show [11] that regular dessins - those for which the group of colourand orientation-preserving automorphisms acts transitively on the edges - on surfaces of genus $g>1$ are almost uniquely determined: their surfaces $C$ are called quasiplatonic, and they can be characterized by the fact that their surface groups $\Gamma$ are normal subgroups of triangle groups $\Delta$. It is nontrivial but not too surprising that the existence of several non-isomorphic regular dessins on the same curve $C$ is always induced by the finitely many and well-known inclusion relations between different triangle groups. 
Regular dessins are in particular uniform, that is (due to the symmetry under the automorphism group) all white vertices have the same valency, and also all black vertices and all faces. Uniform dessins can be characterized by the fact that the surface groups $\Gamma$ of their surfaces $C$ are contained in triangle groups $\Delta$, but they are no longer necessarily normal subgroups; the signature $(r, s, t)$ of $\Delta$ is determined by the valencies of the dessin. In the terminology of point (3) of the above enumeration, the uniform dessins correspond to inclusions $\Gamma<\Delta$ with torsion-free subgroup $\Gamma$. In this case, inclusions between triangle groups always induce new uniform dessins, so one may ask the more interesting question of when and how many different uniform dessins of the same signature may exist on one curve. The answer is given in [10]: an unexpected large number of different uniform dessins of the same signature exists if and only if $\Gamma$ is contained in a congruence subgroup of an arithmetic triangle group $\Delta$, for details see Section 3.

The present paper is in some sense a continuation of [10]. We determine fields of moduli and some fields of definition of the curves involved and of these exotic uniform dessins - at least in the case of Shimura curves whose surface groups are principal congruence subgroups $\Gamma=\Delta\left(\mathfrak{p}^{n}\right)$ of prime power level in an arithmetic triangle group $\Delta$.

\section{Fields of moduli and fields of definition}

Let $S$ be a smooth algebraic curve and $k \subseteq \mathbb{C}$ a field. We say that $k$ is a field of definition of $S$ if there exist homogeneous polynomials

$$
F_{j} \in k\left[x_{0}, x_{1}, \ldots, x_{n}\right], \quad j=1, \ldots, J
$$

such that for $F:=\left\{F_{1}, \ldots, F_{J}\right\}, S$ and

$$
S_{F}=\left\{\left[x_{0}, x_{1}, \ldots, x_{n}\right] \in \mathbb{P}^{n}(\mathbb{C}): F_{j}\left(x_{0}, \ldots, x_{n}\right)=0, \text { for } j=1, \ldots, J\right\}
$$

are isomorphic. Throughout the paper we will deal with Belyi curves, and hence we may always assume that $k<\overline{\mathbb{Q}}$.

Consider now a compact Riemann surface $S$, an algebraic model $S_{F} \cong S$ with $F=$ $\left\{F_{1}, \ldots, F_{J} \in \overline{\mathbb{Q}}\left[x_{0}, \ldots, x_{n}\right]\right\}$ and a Galois element $\sigma \in \operatorname{Gal}(\overline{\mathbb{Q}}):=\operatorname{Gal}(\overline{\mathbb{Q}} / \mathbb{Q})$. One can construct the Galois conjugate curve $S_{F}^{\sigma}=S_{F^{\sigma}}$, where $F^{\sigma}$ is obtained from $F$ by applying $\sigma$ to all coefficients of all $F_{j}$, and the inertia group

$$
I_{S}=\left\{\sigma \in \operatorname{Gal}(\overline{\mathbb{Q}}): S_{F}^{\sigma} \cong S_{F}\right\}
$$

which clearly does not depend on the choice of model. The fixed field

$$
\overline{\mathbb{Q}}^{I_{S}}=\operatorname{Fix}\left(I_{S}\right)=\left\{\alpha \in \overline{\mathbb{Q}}: \sigma(\alpha)=\alpha, \text { for all } \sigma \in I_{S}\right\}
$$

is called the field of moduli of $S$, and we will denote it by $M(S)$. It is a well-known fact that the field of moduli is always contained in any field of definition, but in general $M(S)$ 
is not a field of definition of $S$, see for example [7] or [17]. However, a quasiplatonic curve can always be defined over its field of moduli ([23]).

Similarly, if $G<$ Aut $S$ is a group of automorphisms of $S$, we will say that $k$ is a field of definition of $(S, G)$ if there exists a model $S_{F}$ defined over $k$ and an isomorphism $\varphi: S_{F} \longrightarrow S$ such that the group of automorphisms $\varphi^{-1} G \varphi<$ Aut $S_{F}$ is also defined over $k$. The inertia group is now defined as

$$
I_{(S, G)}=\left\{\sigma \in \operatorname{Gal}(\overline{\mathbb{Q}}): \begin{array}{l}
\text { there exists an isomorphism } f_{\sigma}: S \longrightarrow S^{\sigma} \\
\text { such that } \alpha^{\sigma} \circ f_{\sigma}=f_{\sigma} \circ \alpha, \forall \alpha \in G
\end{array}\right\}
$$

in other words, for every $\sigma \in I_{(S, G)}$ there is an isomorphism $f_{\sigma}: S \longrightarrow S^{\sigma}$ such that the diagram

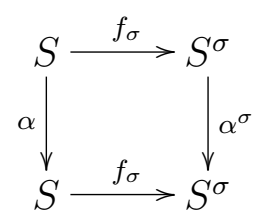

is commutative for every $\alpha \in G$. Accordingly, the field of moduli of the pair $(S, G)$ is $M(S, G)=\overline{\mathbb{Q}}^{I_{(S, G)}}$.

Consider now a Belyi function $\beta: S \longrightarrow \mathbb{P}^{1}(\mathbb{C})$ defining a dessin d'enfant $\mathcal{D}$ on $S$. The group of automorphisms of $\beta$ is defined as the subgroup of $\operatorname{Aut} S$ given by $\operatorname{Aut}(S, \beta)=$ $\{f \in \operatorname{Aut} S: \beta=\beta \circ f\}$. We will write $\operatorname{AutD}:=\operatorname{Aut}(S, \beta)$. For every model $S_{F}$ as above $\beta \circ \varphi$ is a rational function on $S_{F}$. We will say that $k$ is a field of definition of $(S, \beta)$ if there exists a model $S_{F}$ of $S$ such that both $F$ and the covering $\beta \circ \varphi$ are defined over $k$. Accordingly, we will define the field of moduli $M(S, \beta)$ as the field fixed by

$$
I_{(S, \beta)}=\left\{\sigma \in \operatorname{Gal}(\overline{\mathbb{Q}}): \begin{array}{l}
\text { there exists an isomorphism } f_{\sigma}: S \longrightarrow S^{\sigma} \\
\text { such that } \beta^{\sigma} \circ f_{\sigma}=\beta
\end{array}\right\} .
$$

We will also call $M(\mathcal{D}):=M(S, \beta)$ the field of moduli of the dessin $\mathcal{D}$ and, correspondingly, a field of definition of $\mathcal{D}$ will be a field of definition for the Belyi pair $(S, \beta)$. Clearly, $I_{(S, \beta)}<I_{S}$ and therefore $M(S)<M(S, \beta)$.

In many important cases these fields of moduli coincide. For instance, the fields of moduli of regular Belyi functions often agree with the field of definition of the surface in which they are defined. Recall that a Belyi function $\beta$ is called regular if it defines a normal covering $S \rightarrow S / G \cong \mathbb{P}^{1}(\mathbb{C})$ for some group of automorphisms $G<$ Aut $S$. In this case the corresponding dessins are also called regular and the surface $S$ is called quasiplatonic.

Lemma 1. If $S$ is quasiplatonic with surface group $\Gamma \triangleleft \Delta$ where $\Delta$ is a maximal triangle group, then we have $M(S)=M(S, \beta)$ for the Belyi function

$$
\beta: \Gamma \backslash \mathbb{H} \rightarrow \Delta \backslash \mathbb{H}
$$

Proof. We may suppose that $S$ is defined over $M(S)$, so $\beta^{\sigma}$ is another regular Belyi function of the same degree on $S$ for all $\sigma \in I_{S}$. But, since $\Delta$ is maximal, one has $\operatorname{Aut}(S, \beta)=$ $\operatorname{Aut} S=\operatorname{Aut}\left(S, \beta^{\sigma}\right)$ and, being $\beta$ and $\beta^{\sigma}$ regular, one has $\beta=\beta^{\sigma}$. 
Remark 1. It is useful to notice what may happen if the triangle group considered is not maximal. Take for example the possibility $r=s \neq t$; then there may exist a $\sigma \in I_{S}$ fixing $S$ but exchanging the zero set of its Belyi function $B$ and the zero set of $1-B$ such that $M(S, B)$ can be a quadratic extension of $M(S)$. The same problem may occur for uniform non-regular Belyi functions, of course.

A refined argument shows how to extend Lemma 1 in some cases to non-maximal triangle groups:

Lemma 2. Let $S$ be a quasiplatonic surface with surface group $\Gamma \triangleleft \Phi$ where $\Phi$ is a nonmaximal triangle group of signature $(r, r, t), r \neq t$, with regular Belyi function $B$. Suppose moreover that $\Gamma$ is also normal in the maximal triangle group $\Delta$ of signature $(2, r, 2 t)$, containing $\Phi$ with index $|\Delta: \Phi|=2$. Then there is a model for $(S, B)$ defined over $M(S)$.

Proof. We can assume $S$ to be defined over $M(S)$, and that all zeros of $B$ and of $1-B$ have order $r$. Any $\sigma \in \operatorname{Gal}(\overline{\mathbb{Q}} / M(S))$ fixes $S$ and either fixes $B$ or maps it to $B^{\sigma}=1-B$, see Remark 1. Both $B$ and $1-B$ take the value $\frac{1}{2}$ at the same points $x \in S$, images of the $\Phi$-orbit (and also $\Delta$-orbit) of the fixed point of the canonical order 2 generator of $\Delta$ under the projection $\mathbb{H} \rightarrow \Gamma \backslash \mathbb{H}$. We take such a point $x \in B^{-1}\left(\frac{1}{2}\right)$; for any $\sigma \in \operatorname{Gal}(\overline{\mathbb{Q}} / M(S))$, also $\sigma(x) \in B^{-1}\left(\frac{1}{2}\right)$. Since it is not a ramification point of $B$ nor $1-B$, there is a unique $g_{\sigma}$ in the covering group $\Phi / \Gamma$ of $B$ (and of $1-B$ ) with the property $g_{\sigma}(x)=\sigma(x)$. Now we distinguish two cases:

First, if $B^{\sigma}=B$, we put $f_{\sigma}:=g_{\sigma} \in$ Aut $S$.

Second, if $B^{\sigma}=1-B$, there is an involution $i_{\sigma} \in$ Aut $S$ induced by an order 2 generator of $\Delta$ fixing $x$, interchanging the zero sets of $B$ and $1-B$ and fixing their pole sets. In this case, we put $f_{\sigma}:=g_{\sigma} \circ i_{\sigma}$.

In both cases, the $f_{\sigma} \in$ Aut $S$ are uniquely determined by $\sigma$ and make the following diagrams commutative:

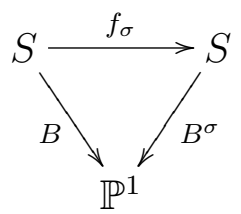

By uniqueness, these $f_{\sigma}$ trivially satisfy Weil's cocycle condition [22], whence $(S, B)$ can also be defined over $M(S)$.

We learned from Rubén Hidalgo another application of Weil's criterion which turns out to be very useful for proving the following theorem - for more sophisticated versions see [5].

Theorem 1. Let $S$ be a quasiplatonic curve of genus $g>1$ with full automorphism group Aut $S=G$. If $G$ contains its centre $Z(G)$ as a direct factor, that is if $G \cong G^{\prime} \times Z(G)$, the pair $(S, G)$ can be defined over its field of moduli $M(S, G)$. 
Proof. We can suppose that $S$ is defined over its field of moduli $M(S)$. Let $\sigma \in I_{(S, G)}$. Every automorphism $f_{\sigma}$ satisfying the condition

$$
f_{\sigma} \circ \alpha \circ f_{\sigma}^{-1}=\alpha^{\sigma} \quad \text { for all } \quad \alpha \in G
$$

must be of the form $\left(g_{\sigma}, h\right) \in G^{\prime} \times Z(G)$ for some $g_{\sigma} \in G^{\prime}$. Then the set $\left\{\left(g_{\sigma}, 1\right)\right\}$ satisfies Weil's cocycle condition and we can define $(S, G)$ over $M(S, G)$.

A remark in the preprint [2] suggests that the result may still be true without the hypothesis about the centre. We will not use such a stronger version since the automorphism groups considered here are of type $\mathrm{PSL}_{2}, \mathrm{PGL}_{2}$ or direct products of such groups with cyclic factors $C_{2}$, so Theorem 1 applies.

Corollary 1. Under the same hypotheses, let $U$ be a subgroup of $G$ and $C:=U \backslash S$ the quotient curve. Then, $C$ can be defined over the field $M(S, G)$. Moreover, all such quotient curves $C$ can be simultaneously defined over $M(S, G)$ in the sense that all their function fields $M(S, G)(C)$ are subfields of $M(S, G)(S)$.

Proof. Recall that $k$ is a field of definition for $C$ if the function field $\mathbb{C}(C)$ can be obtained by a constant field extension of a function field $k(C)$, that is tensoring some ring of functions $k[C]$ with $\mathbb{C}$ and taking the quotient field. Now, we know that the function field of $S$ is a constant field extension of $M(S, G)(S)$ and that moreover all automorphisms of $S$ are defined over $M(S, G)$. Therefore, $M(S, G)(S)$ is a Galois extension of a rational function field $M(S, G)(\beta)$, and $M(S, G)(C)$ is the fixed field of the subgroup $U$. This construction works simultaneously for all quotient curves of $S$.

\section{Congruence subgroups and uniform dessins}

A finite group $G$ is called a Hurwitz group if it acts as automorphism group on a compact Riemann surface $S$ of genus $g>1$ and has order $84(g-1)$, which is Hurwitz's universal upper bound for all such automorphism group orders. In this case the surface is moreover quasiplatonic: it is well known that $S$ is uniformised in this case by a normal subgroup $K$ of the triangle group $\Delta(2,3,7)$ and, in particular, one has $G \cong \Delta(2,3,7) / K$. A classical theorem by Macbeath [15] shows that $\operatorname{PSL}\left(2, \mathbb{F}_{q}\right)$ is a Hurwitz group exactly in the following cases

(i) $q=7$,

(ii) $q=p$ prime for $p \equiv \pm 1 \bmod 7$,

(iii) $q=p^{3}$ for $p$ prime and $p \equiv \pm 2$ or $\pm 3 \bmod 7$.

The corresponding Riemann surfaces are usually known as Macbeath-Hurwitz curves and the first examples are found in genus three (Klein's Riemann surface, with automorphism 
group isomorphic to $\operatorname{PSL}\left(2, \mathbb{F}_{7}\right)$ ), genus seven (the Fricke-Macbeath curve with automorphism group $\operatorname{PSL}\left(2, \mathbb{F}_{8}\right)$ ) and genus fourteen (where there exist three non-isomorphic but Galois conjugate Macbeath-Hurwitz curves with automorphism group isomorphic to $\left.\operatorname{PSL}\left(2, \mathbb{F}_{13}\right)\right)$.

It was proved in [6] by A. Džambić that all Macbeath-Hurwitz curves can be constructed arithmetically as follows. Given a number field $k$, denote by $\mathcal{O}_{k}$ its ring of integers. The triangle group $\Delta(2,3,7)$ is the norm 1 group of a maximal order in a quaternion algebra $A$ over the field $k=\mathbb{Q}(\cos \pi / 7)$ (more precisely its image under the canonical homomorphism $\operatorname{SL}(2, \mathbb{R}) \rightarrow \operatorname{PSL}(2, \mathbb{R}))$, and it can be seen as a subgroup of $\operatorname{PSL}\left(2, \mathcal{O}_{L}\right)$, the projective group of determinant 1 matrices over the ring of integers $\mathcal{O}_{L}$ of an at most quadratic extension $L$ of $k$. Any rational prime $p$ defines an ideal $p \mathcal{O}_{k}$ in $\mathcal{O}_{k}$ such that

(i) if $p=7$ then $p$ is ramified and $p \mathcal{O}_{k}=\mathfrak{p}^{3}$ for a prime ideal $\mathfrak{p} \subset \mathcal{O}_{k}$ of norm $q=$ $N(\mathfrak{p})=7$;

(ii) if $p \equiv \pm 1 \bmod 7$ then $p$ splits, i.e. $p \mathcal{O}_{k}=\mathfrak{p}_{1} \mathfrak{p}_{2} \mathfrak{p}_{3}$ for prime ideals $\mathfrak{p}_{1}, \mathfrak{p}_{2}, \mathfrak{p}_{3} \subset \mathcal{O}_{k}$ of norm $q=N\left(\mathfrak{p}_{i}\right)=p$;

(iii) if $p \equiv \pm 2$ or $\pm 3 \bmod 7$ then $p$ is inert, i.e. $p \mathcal{O}_{k}$ is a prime ideal in $\mathcal{O}_{k}$ of norm $q=N(\mathfrak{p})=p^{3}$.

For every prime $\mathfrak{p}$ in $\mathcal{O}_{k}$ we can define the subgroup of matrices of $\Delta(2,3,7)$ congruent to the identity modulo $\mathfrak{p}$. This is a normal torsion-free subgroup of $\Delta(2,3,7)$ with quotient group isomorphic to $\operatorname{PSL}\left(2, \mathbb{F}_{q}\right)$ where $q=N(\mathfrak{p})$, yielding therefore a Macbeath-Hurwitz curve.

\subsection{Principal congruence subgroups}

One can generalise the construction above in the following way. Consider any arithmetic triangle group $\Delta=\Delta(r, s, t)$ which is the norm 1 group $\mathcal{M}^{1}$ of a maximal order $\mathcal{M}$ of a quaternion algebra $A$ over a field $k$. In the last section, we will extend our assumptions to triangle groups containing (always with index 2 or 4 ) such a norm 1 group. Up to an extension of the quotient groups $\operatorname{PSL}\left(2, \mathbb{F}_{q}\right)$ to $\mathrm{PGL}\left(2, \mathbb{F}_{q}\right)$ and/or adding one or two direct factors $C_{2}$, most results of the present section remain valid. It is well known [20] that in the case of arithmetic triangle groups, the invariant trace field $k$ has always class number 1 , and therefore any prime ideal in $\mathcal{O}_{k}$ is principal, hence of the form $\mathfrak{p}=\pi \mathcal{O}_{k}$ for some prime $\pi \in \mathcal{O}_{k}$. Given such a prime $\mathfrak{p}$ in $\mathcal{O}_{k}$ one can define the local quaternion algebra $A_{\mathfrak{p}}$ over the $\mathfrak{p}$-adic field $k_{\mathfrak{p}}$.

For each prime $\mathfrak{p}$ not dividing the discriminant of $A$, the $\mathfrak{p}$-adic completion $A_{\mathfrak{p}}$ is isomorphic to $M_{2}\left(k_{\mathfrak{p}}\right)$. As in the previous case one can define the principal congruence subgroup of level $\mathfrak{p}$. It is defined as the (normal) subgroup $\Delta(\mathfrak{p})$ of $\Delta$ whose localisation with respect to a prime $\mathfrak{q} \in \mathcal{O}_{k}$ coincides with

$$
\Phi(\mathfrak{p})=\left\{\left(\begin{array}{ll}
a & b \\
c & d
\end{array}\right) \in \mathcal{M}_{\mathfrak{q}} \cong M_{2}\left(\mathcal{O}_{\mathfrak{q}}\right):\left(\begin{array}{ll}
a & b \\
c & d
\end{array}\right) \equiv\left(\begin{array}{ll}
1 & 0 \\
0 & 1
\end{array}\right) \bmod \mathfrak{q}\right\}
$$


(here the congruences have to be read coefficient-wise $\bmod \pi \mathcal{O}_{\mathfrak{q}}$ ) if $\mathfrak{q}=\mathfrak{p}$ and with $\mathcal{M}_{\mathfrak{q}}^{1}$ otherwise, where $\mathcal{M}_{\mathfrak{p}}$ stands for the localisation of $\mathcal{M}$ and $\mathcal{O}_{\mathfrak{q}}$ denotes the ring of integers of the local field $k_{\mathfrak{q}}$. The existence and uniqueness of such a subgroup is granted by the strong approximation theorem for arithmetic groups (see for example [21] or [14]). We have $\Delta / \Delta(\mathfrak{p}) \cong \operatorname{PSL}\left(2, \mathbb{F}_{q}\right)$ where $q=N(\mathfrak{p}):=\left|\mathcal{O}_{k} / \mathfrak{p}\right|$.

The first consequence is that all these surfaces $S=\Delta(\mathfrak{p}) \backslash \mathbb{H}$ have a regular Belyi function $\beta: \Delta(\mathfrak{p}) \backslash \mathbb{H} \longrightarrow \Delta \backslash \mathbb{H}$, given by the normal inclusion $\Delta(\mathfrak{p}) \triangleleft \Delta$, and an automorphism group of order $\mid$ Aut $S|=| N(\Delta(\mathfrak{p})) / \Delta(\mathfrak{p})|\geq| \operatorname{PSL}\left(2, \mathbb{F}_{q}\right) \mid$ where $N$ denotes the normaliser in $\operatorname{PSL}(2, \mathbb{R})$.

Analogously, for each positive integer $n$ we can consider the principal congruence subgroup $\Delta\left(\mathfrak{p}^{n}\right)$ of level $\mathfrak{p}^{n}$, whose localisation in $\mathfrak{p}$ corresponds to

$$
\Phi\left(\mathfrak{p}^{n}\right)=\left\{\left(\begin{array}{ll}
a & b \\
c & d
\end{array}\right) \in \mathcal{M}_{\mathfrak{p}} \cong M_{2}\left(\mathcal{O}_{\mathfrak{p}}\right):\left(\begin{array}{ll}
a & b \\
c & d
\end{array}\right) \equiv\left(\begin{array}{ll}
1 & 0 \\
0 & 1
\end{array}\right) \bmod \mathfrak{p}^{n}\right\}
$$

This time the quotient $\Delta / \Delta\left(\mathfrak{p}^{n}\right)$ is isomorphic to $\operatorname{PSL}\left(2, \mathcal{O}_{\mathfrak{p}} / \mathfrak{p}^{n} \mathcal{O}_{\mathfrak{p}}\right)$. Once again the normal inclusion $\Delta\left(\mathfrak{p}^{n}\right) \triangleleft \Delta$ yields a regular Belyi function on $S=\mathbb{H} / \Delta\left(\mathfrak{p}^{n}\right)$.

¿From the point of view of quaternion algebras, principal congruence subgroups of level $\mathfrak{p}^{n}$ with $n \geq 1$ correspond to the intersection of certain maximal orders in the local quaternion algebra $A_{\mathfrak{p}}$. More precisely, maximal orders in a split local quaternion algebra $A_{\mathfrak{p}}$ can be represented as vertices of a regular Bruhat-Tits-tree of valency $q+1$, where $q=N(\mathfrak{p})$ (see Figure 1). The principal congruence subgroup of level $\mathfrak{p}^{n}$ corresponds then to the intersection of all the vertices at distance $\leq n$ from some point, which represents the maximal order $\mathcal{M}$ whose norm 1 group is $\Delta$.

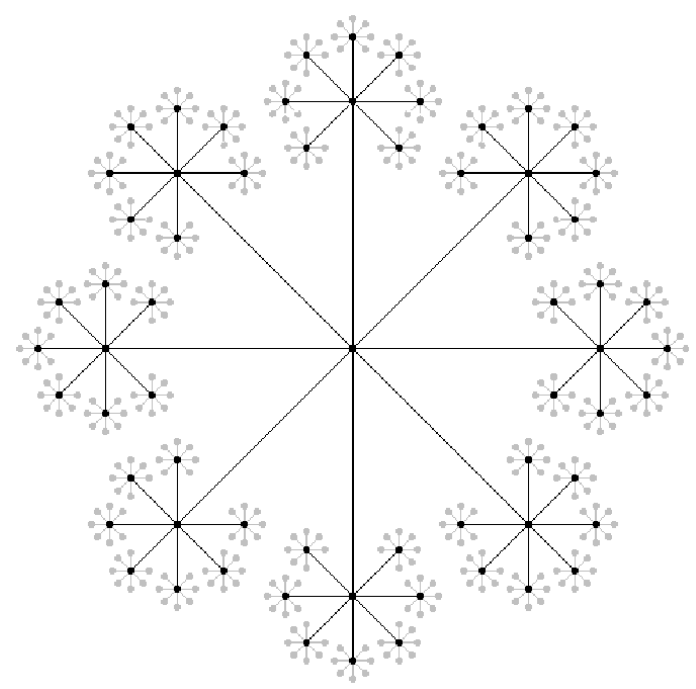

Figure 1: Part of the tree of maximal orders of a local algebra $A_{\mathfrak{p}}$ corresponding to $\Delta\left(\mathfrak{p}^{3}\right)$ (in the case $N(\mathfrak{p})+1=8$ ). 


\subsection{Multiple uniform dessins}

In [10] we studied under which conditions a surface $S$ contains different uniform Belyi functions of a given type $(r, s, t)$. This is equivalent to determine when the uniformising group $K$ of $S$ is contained in different triangle groups of that signature. In the case when $\Delta=\Delta(r, s, t)$ is the norm 1 group of a maximal order in a quaternion algebra as above, this happens if and only if $K$ is contained in a group conjugate in $\Delta$ to the congruence subgroup $\Delta_{0}(\mathfrak{p})$, where $\mathfrak{p}$ is a prime in $\mathcal{O}_{k}$ not dividing the discriminant of $A$, and $\Delta_{0}(\mathfrak{p})$ is defined as the subgroup of $\Delta$ whose localisation with respect to the prime $\mathfrak{p}$ is

$$
\Phi_{0}(\mathfrak{p})=\left\{\left(\begin{array}{ll}
a & b \\
c & d
\end{array}\right) \in \mathcal{M}_{\mathfrak{p}}^{1} \cong \operatorname{SL}\left(2, \mathcal{O}_{\mathfrak{p}}\right): c \equiv 0 \bmod \mathfrak{p}\right\}
$$

Remark 2. Even if $\Delta$ is strictly larger than the norm 1 group of a maximal order (by index 2 or 4 , as already mentioned) we define the congruence subgroups $\Delta(\mathfrak{p})$ and $\Delta_{0}(\mathfrak{p})$ always as subgroups only consisting of norm 1 elements. The same convention will be used for higher power levels $\mathfrak{p}^{j}$.

In order to explain this multiple inclusion of $K$ in different conjugate triangle groups one has to introduce the so called Fricke (or Atkin-Lehner) extension $\Delta_{\mathrm{Fr}}(\mathfrak{p})$. In the local algebra $A_{\mathfrak{p}}$, the group $\Phi_{0}(\mathfrak{p})$ is the norm 1 group of an Eichler order $\mathcal{E}_{\mathfrak{p}}$, that is the intersection of two maximal orders $\mathcal{M}_{\mathfrak{p}}$ and $\gamma^{-1} \mathcal{M}_{\mathfrak{p}} \gamma$, where $\gamma=\left(\begin{array}{ll}\pi & 0 \\ 0 & 1\end{array}\right)$. The norm 1 groups of these two (local) maximal orders correspond in the global case to two triangle groups $\Delta=\Delta_{1}$ and $\Delta_{2}$ of the same type. The (Fricke) element $\left(\begin{array}{cc}0 & \pi^{-1} \\ -1 & 0\end{array}\right) \in A_{\mathfrak{p}}$ conjugates one maximal order into the other, and therefore induces an isomorphism of $\Phi_{0}(\mathfrak{p})$ of order two, called the Fricke involution. In the global case, this Fricke element corresponds to a matrix in $\operatorname{GL}(2, \mathbb{R})$ interchanging $\Delta_{1}$ and $\Delta_{2}$ by conjugation. By the rigidity of triangle groups, this conjugation can be realised inside $\operatorname{PSL}(2, \mathbb{R})$ by an element $\alpha_{\mathfrak{p}}$, which generates an index two extension $\Delta_{\mathrm{Fr}}(\mathfrak{p})=\left\langle\Delta_{0}(\mathfrak{p}), \alpha_{\mathfrak{p}}\right\rangle$ called the Fricke extension.

Since $\alpha_{\mathfrak{p}}$ normalises $\Delta_{0}(\mathfrak{p})$, but not $\Delta$, and $\alpha_{\mathfrak{p}}^{2} \in \Delta_{0}(\mathfrak{p})$, conjugation by $\alpha_{\mathfrak{p}}$ induces an involution on the curve $\Delta_{0}(\mathfrak{p}) \backslash \mathbb{H}$. We call it therefore the Fricke involution for $\Delta_{0}(\mathfrak{p})$ (even if it might not satisfy $\alpha_{\mathfrak{p}}^{2}=1$ in $\left.\operatorname{PSL}(2, \mathbb{R})\right)$. As a consequence every group $K<\Delta_{0}(\mathfrak{p})$ is included in both $\Delta$ and $\Delta_{2}=\alpha_{\mathfrak{p}} \Delta \alpha_{\mathfrak{p}}^{-1}$, yielding two different uniform dessins in $K \backslash \mathbb{H}$.

In the same way, for every integer $j>1$ one can introduce the congruence group $\Delta_{0}\left(\mathfrak{p}^{j}\right)$ which corresponds in the local algebra $A_{\mathfrak{p}}$ to

$$
\Phi_{0}\left(\mathfrak{p}^{j}\right)=\left\{\left(\begin{array}{ll}
a & b \\
c & d
\end{array}\right) \in \mathrm{SL}\left(2, \mathcal{O}_{\mathfrak{p}}\right): c \equiv 0 \bmod \mathfrak{p}^{j}\right\} .
$$

Note that $\Delta_{0}\left(\mathfrak{p}^{j}\right)<\Delta_{0}(\mathfrak{p})$. The Fricke involution is in this case an element $\alpha_{\mathfrak{p}^{j}}$ corresponding in the local case to the element

$$
\left(\begin{array}{cc}
0 & \pi^{-j} \\
-1 & 0
\end{array}\right)
$$


and $\Delta_{0}\left(\mathfrak{p}^{j}\right)$ is normal in the Fricke extension $\Delta_{\mathrm{Fr}}\left(\mathfrak{p}^{j}\right)=\left\langle\Delta_{0}\left(\mathfrak{p}^{j}\right), \alpha_{\mathfrak{p}^{j}}\right\rangle$. However, none of the groups $\Delta_{0}\left(\mathfrak{p}^{l}\right)$ is normal in $\Delta_{\mathrm{Fr}}\left(\mathfrak{p}^{j}\right)$, for $j<l$, and neither is any of its $\Delta$-conjugates $\Delta_{0}^{i}\left(\mathfrak{p}^{l}\right)$. Existence and uniqueness of all these Fricke involutions is well known in the case of the elliptic modular group $\Delta(2,3, \infty)=\operatorname{PSL}(2, \mathbb{Z})$ and is probably known to the experts in the cases we need here. However, by lack of a good reference we include a statement and a proof:

Lemma 3. Let $\Delta$ be an arithmetic Fuchsian triangle group, a PSL-image of a norm 1 group of a maximal order $M_{0}$ in the quaternion algebra $A$ with totally real centre field $k$ of class number 1 , and $\mathfrak{p}$ a prime ideal in $k$ not dividing the discriminant of $A$. We consider $A$ as embedded in the matrix algebra $M_{2}(\mathbb{R})$. For all $j \geq 1$, the congruence subgroup $\Delta_{0}\left(\mathfrak{p}^{j}\right)$ is the norm 1 subgroup of the intersection $M_{0} \cap M_{j}$ of two maximal orders $M_{0}$ and $M_{j}$ in $A$. These two maximal orders are conjugate under a (Fricke) involution $\alpha_{\mathfrak{p} j}$; we can suppose that - as an element of $A-\alpha_{\mathfrak{p}^{j}}$ has norm $\pi^{-j}$. Then the class $\alpha_{\mathfrak{p}^{j}} \Delta_{0}\left(\mathfrak{p}^{j}\right)$ is uniquely determined.

Proof. Since we are considering only congruence subgroups of levels $\mathfrak{p}^{j}$, we can see the maximal orders as intersections of their $\mathfrak{p}$-adic completions with $A$ and visualise the local maximal orders by the vertices of the Bruhat-Tits tree already introduced above, see Figure 1.

For the first claim about the role of $\Delta_{0}\left(\mathfrak{p}^{j}\right)$ as norm 1 subgroup of the intersection $M_{0} \cap M_{j}$ of two maximal orders $M_{0}$ and $M_{j}$ one may consult [10] to see that these orders correspond to two vertices which are at distance $j$ from each other in the Bruhat-Tits tree.

Existence of $\alpha_{\mathfrak{p}^{j}}$ : since $k$ has class number 1 , all maximal orders are conjugate in $A$ [21], so we have some $\gamma \in A^{*}$ such that $M_{j}=\gamma M_{0} \gamma^{-1}$. It is easy to see that we may even suppose $\gamma \in \mathrm{SL}(2, \mathbb{R})$. If $M_{0}=\gamma M_{j} \gamma^{-1}$, the element $\gamma^{2}$ fixes both $M_{0}$, $M_{j}$, hence belongs to $\Delta_{0}\left(\mathfrak{p}^{j}\right)$, and $\gamma$ normalises this subgroup. If not, we have $\gamma M_{j} \gamma^{-1}=M^{\prime} \neq M_{0}$, both having distance $j$ from $M_{j}$. But in this case there is some $\delta$ in the norm 1 group of $M_{j}$ (hence fixing $M_{j}$ ) and sending $M^{\prime}$ to $M_{0}$, see the arguments in the proof of the next lemma. Then, $\delta \gamma$ exchanges $M_{0}$ and $M_{j}$, so we can consider this element as $\alpha_{\mathfrak{p}^{j}}$.

Uniqueness: suppose we have two such elements $\gamma, \delta \in \operatorname{PSL}(2, \mathbb{R})$ exchanging $M_{0}$ and $M_{j}$ under conjugation. They extend to automorphisms of $A$, so we can assume by the Skolem-Noether Theorem that they are PSL-images of elements of $A$. Then $\delta^{-1} \gamma$ fixes $M_{0}$ and $M_{j}$ and - as an element of $A$ - has norm 1 , hence belongs to $\Delta_{0}\left(\mathfrak{p}^{j}\right)$ and induces the identity on the quotient curve $\Delta_{0}\left(\mathfrak{p}^{j}\right) \backslash \mathbb{H}$.

Now, the principal congruence subgroups $\Delta\left(\mathfrak{p}^{n}\right)$ are obviously included in $\Delta_{0}\left(\mathfrak{p}^{j}\right)$ for each $j=1, \ldots, n$, hence we deduce that, in particular, the corresponding surfaces $\Delta\left(\mathfrak{p}^{n}\right) \backslash \mathbb{H}$ contain several uniform dessins. In fact, one has the following

Lemma 4. Under the same hypothesis as in Lemma 3, for each $j=1, \ldots, n$ there are $q^{j-1}(q+1)$ congruence subgroups $\Delta_{0}^{i}\left(\mathfrak{p}^{j}\right)$ conjugate to $\Delta_{0}\left(\mathfrak{p}^{j}\right)$ in $\Delta$, for $i=0, \ldots, q^{j-1}(q+1)-1$. Each of them is contained in $\Delta$ and in $j$ different triangle groups conjugate to $\Delta$, in which $\Delta\left(\mathfrak{p}^{n}\right)$ is included non-normally. Every $\Delta_{0}^{i}\left(\mathfrak{p}^{j}\right)$ is the intersection 
of $\Delta$ with a conjugate triangle group $\Delta^{j, i}$, and for fixed $j$, the different $\Delta^{j, i}$ form an orbit under conjugation by $\Delta$.

Proof. The proof proceeds by induction on $j$. The group $\Delta_{0}(\mathfrak{p})$ is an index $q+1$ subgroup of $\Delta$, and for each class of elements $\rho_{i} \in \Delta$ modulo $\Delta_{0}(\mathfrak{p}), i=0, \ldots, q$, we can construct the group $\Delta_{0}^{i}(\mathfrak{p}) \equiv \rho_{i} \cdot \Delta_{0}(\mathfrak{p}) \cdot \rho_{i}^{-1}$ for $\rho_{0}=\mathrm{Id}, \ldots, \rho_{q}$, such that

$$
\Delta\left(\mathfrak{p}^{n}\right) \triangleleft \Delta_{0}^{i}(\mathfrak{p})<\Delta
$$

For each of them we have the Fricke involution $\alpha_{i}:=\rho_{i} \alpha_{\mathfrak{p}} \rho_{i}^{-1}$, the Fricke extension $\Delta_{\mathrm{Fr}}^{i}(\mathfrak{p})=$ $\left\langle\Delta_{0}^{i}(\mathfrak{p}), \alpha_{i}\right\rangle \nless \Delta$ and therefore a non-normal inclusion of $\Delta\left(\mathfrak{p}^{n}\right)$ in $\alpha_{i} \Delta \alpha_{i}^{-1}$, yielding the following diagram of inclusions

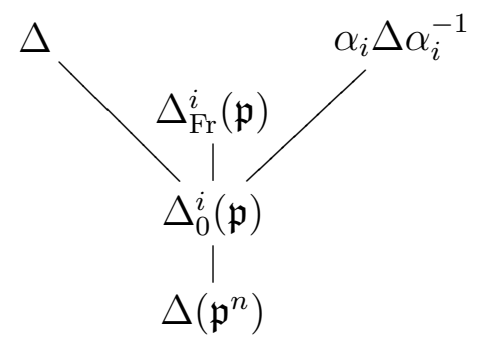

Now suppose that there are $q^{j-1}(q+1)$ congruence subgroups conjugate to $\Delta_{0}\left(\mathfrak{p}^{j}\right)$ in $\Delta$. Inside this subgroup lies $\Delta_{0}\left(\mathfrak{p}^{j+1}\right)$ with index $q$. Its normaliser in $\Delta_{0}\left(\mathfrak{p}^{j}\right)$ is trivial, and therefore there are $q$ subgroups of $\Delta_{0}\left(\mathfrak{p}^{j}\right)$ conjugate to $\Delta_{0}\left(\mathfrak{p}^{j+1}\right)$. Now the Fricke element $\alpha_{\mathfrak{p}^{j+1}}$ conjugates $\Delta$ into a different triangle group $\Delta^{j, 1}$ in which $\Delta\left(\mathfrak{p}^{n}\right)$ is included.

The claim about the conjugacy of the different $\Delta_{0}^{i}\left(\mathfrak{p}^{j}\right)$ for fixed $j$ follows from the action of $\Delta$ on the fake projective line $\mathbb{P}^{1}\left(\mathcal{O}_{k} / \mathfrak{p}^{j}\right)$ consisting of pairs $(x, y) \in\left(\mathcal{O}_{k} / \mathfrak{p}^{j}\right)^{2}$, not both coordinates divisible by $\mathfrak{p}$, modulo the diagonal action of $\left(\mathcal{O}_{k} / \mathfrak{p}^{j}\right)^{*}$. The action of $\Delta$ on this fake projective line is transitive again because the $\mathfrak{p}$-adic completion of $\Delta$ is isomorphic to the $\operatorname{PSL}\left(2, \mathcal{O}_{\mathfrak{p}}\right)$ of the $\mathfrak{p}$-adic integers in $k_{\mathfrak{p}}$, and the subgroups $\Delta_{0}^{i}\left(\mathfrak{p}^{j}\right)$ are the stabiliser subgroups of the different points on this fake projective line. They are obviously different and conjugate under the action of $\Delta$ by transitivity. By the same conjugations, $\Delta$ acts on the $\Delta^{j, i}$ giving the final claim.

The representation in the tree of local maximal orders is the following. The $q+1$ groups $\Delta_{0}^{i}(\mathfrak{p})$ correspond to the $q+1$ paths joining the middle vertex with each of its neighbours at distance 1 , which correspond to the $q+1$ groups $\alpha_{i} \Delta \alpha_{i}^{-1}$. Similarly, for each $j=2, \ldots, n$ the groups $\Delta_{0}^{i}\left(\mathfrak{p}^{j}\right), i=1, \ldots, q^{j-1}(q+1)$ are represented by the paths joining the middle vertex with each of its distance $j$ neighbours, which correspond precisely to the triangle groups mentioned in the previous lemma.

Example 1. The principal congruence subgroup $\Delta\left(\mathfrak{p}_{7}\right)<\Delta(2,3,7)$, for a prime $\mathfrak{p}_{7}$ in $\mathbb{Q}(\cos \pi / 7)$ dividing the rational prime 7 , uniformises Klein's quartic $\mathcal{K}$ and, accordingly, one has one regular Belyi function and $N\left(\mathfrak{p}_{7}\right)+1=8$ uniform dessins of type $(2,3,7)$ on $\mathcal{K}$. 
Example 2. Consider Bring's curve $\mathcal{B}$, given by the equations

$$
\left\{\begin{array}{l}
x_{0}+x_{1}+x_{2}+x_{3}+x_{4}=0 \\
x_{0}^{2}+x_{1}^{2}+x_{2}^{2}+x_{3}^{2}+x_{4}^{2}=0 \\
x_{0}^{3}+x_{1}^{3}+x_{2}^{3}+x_{3}^{3}+x_{4}^{3}=0
\end{array}\right.
$$

in $\mathbb{P}^{4}(\mathbb{C})$. It is known that $\mathcal{B}$ is the only Riemann surface of genus 4 admitting the symmetric group on 5 elements $\mathfrak{S}_{5}$ as a group of automorphisms (see for example [16]), and that this action is simply given by permutation of the projective coordinates.

Now, the triangle group $\Delta:=\Delta(2,5,5)$ is the norm 1 group of a maximal order in a quaternion algebra defined over $\mathbb{Q}(\sqrt{5})$, and the principal congruence subgroup $\Delta(\sqrt{5}) \triangleleft$ $\Delta(2,5,5)$ uniformises a surface of genus 4 . This group is also normal in the maximal triangle group $\Delta(2,4,5)$, and it can be proved that $\Delta(2,4,5) / \Delta(\sqrt{5})=\operatorname{PGL}\left(2, \mathbb{F}_{5}\right) \cong \mathfrak{S}_{5}$. As a consequence the surface $\mathcal{B}$ has the principal congruence subgroup $\Delta(\sqrt{5})$ as uniformising group.

By the results in the previous sections, it has a regular Belyi function and $N(\sqrt{5})+1=6$ subgroups $\Delta_{0}^{i}(\sqrt{5})$. Note that in this case, these subgroups lead to 12 uniform Belyi functions of type $(2,5,5)$ on $\mathcal{B}$, if we take into account the renormalizations $B_{i}$ and $1-B_{i}$ as in Remark 1 and Lemma 2.

In the following, we will always suppose that $\Delta$ is an arithmetic Fuchsian triangle group containing a PSL-image of a norm 1 group of a maximal order $M_{0}$ in the quaternion algebra $A$ with totally real centre field.

We will first focus our attention on principal congruence subgroups of level $\mathfrak{p}$. By the discussion above, there are $q+1$ surfaces $S_{i} \cong \mathbb{H} / \Delta_{0}^{i}(\mathfrak{p}), i=0, \ldots, q$, and for each of them there are uniform Belyi functions

$$
\begin{aligned}
& r_{i}: \Delta_{0}^{i}(\mathfrak{p}) \backslash \mathbb{H} \quad \longrightarrow \quad \Delta \backslash \mathbb{H} \\
& u_{i}: \Delta_{0}^{i}(\mathfrak{p}) \backslash \mathbb{H} \quad \longrightarrow \quad \alpha_{i} \Delta \alpha_{i}^{-1} \backslash \mathbb{H}
\end{aligned}
$$

such that $\beta$ and all $\beta_{i}$ decompose via the intermediate coverings $\tau_{i}: S \cong \Delta(\mathfrak{p}) \backslash \mathbb{H} \longrightarrow S_{i} \cong \Delta_{0}^{i}(\mathfrak{p}) \backslash \mathbb{H}$ as

$$
\beta=r_{i} \circ \tau_{i}, \quad \beta_{i}=u_{i} \circ \tau_{i} .
$$

So, for each $i=0, \ldots, q$ one has the following diagram

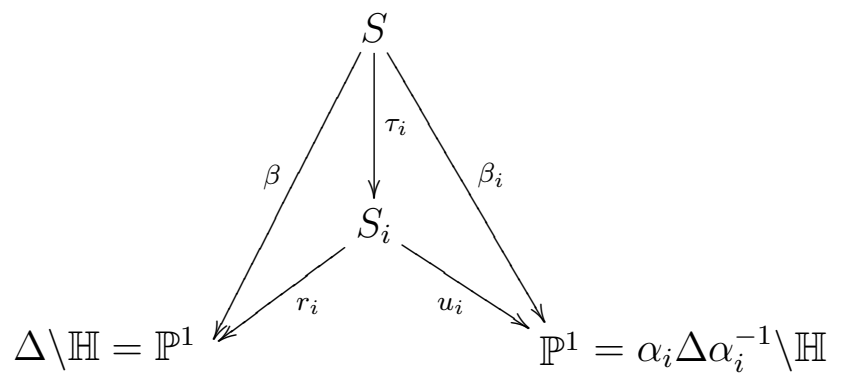


Lemma 5. Let $a_{i}$ be the automorphism of the intermediate curve $S_{i}:=\Delta_{0}^{i}(\mathfrak{p}) \backslash \mathbb{H}$ induced by the Fricke involution $\alpha_{i}$. Then, $u_{i} \circ a_{i}=r_{i}$, hence $\beta=r_{i} \circ \tau_{i}=u_{i} \circ a_{i} \circ \tau_{i}$ and $\beta_{i}=u_{i} \circ \tau_{i}=r_{i} \circ a_{i} \circ \tau_{i}$.

Proof. Remember that $r_{i}$ and $u_{i}$ are the Belyi functions on $S_{i}$ induced by the Belyi functions $\beta$ and $\beta_{i}$ on $S$, and that their dessins $\mathcal{D}$ and $\mathcal{D}_{i}$ come from the tessellations of $\mathbb{H}$ by the fundamental domains $F, F_{i}$ for $\Delta$ and $\alpha_{i} \Delta \alpha_{i}^{-1}$. Since for $F_{i}$ we can take $\alpha_{i}(F)$, the Belyi functions are linked as indicated.

Lemma 5 generalises to higher levels as follows.

Lemma 6. Let $S_{j, i}:=\Delta_{0}^{i}\left(\mathfrak{p}^{j}\right) \backslash \mathbb{H}, \tau_{j, i}$ the quotient map $S_{\mathfrak{p}^{n}} \rightarrow S_{j, i}$ and

$$
r_{j, i}: S_{j, i} \rightarrow \Delta \backslash \mathbb{H}, \quad u_{j, i}: S_{j, i} \rightarrow \alpha_{j, i} \Delta \alpha_{j, i}^{-1} \backslash \mathbb{H}=\Delta^{j, i} \backslash \mathbb{H}
$$

the two quotient maps giving the regular and the nonregular uniform Belyi functions

$$
\beta=r_{j, i} \circ \tau_{j, i} \quad, \quad \beta_{j, i}=u_{j, i} \circ \tau_{j, i}: S_{\mathfrak{p}^{n}} \rightarrow \mathbb{P}^{1}(\mathbb{C}) .
$$

Then the involution $a_{j, i}$ induced by $\alpha_{j, i}$ on $S_{j, i}$ satisfies

$$
u_{j, i}=r_{j, i} \circ a_{j, i} \quad \text { and } \quad r_{j, i}=u_{j, i} \circ a_{j, i} .
$$

\section{Shimura congruence curves of prime power level}

In this section we will get some information about the fields of moduli and the fields of the definition of the curves and dessins described in Section 3.

\subsection{Fields of moduli}

In order to determine explicitly the field of moduli $M\left(S_{\mathfrak{p}}\right)$ of our Shimura curves we could rely on Clark and Voight's Theorem A and Proposition 5.1 in [2], at least in the case of prime levels. We will however prove parts of these results in another way, more similar to the methods already used here.

First we need the following useful lemma.

Lemma 7. Let $\Delta$ be an arithmetically defined cocompact triangle group, containing the norm 1 group of a maximal order of a quaternion algebra $A$ with centre field $k$, and let $\mathfrak{p}$ be a prime ideal in $k$ not dividing the discriminant of $A$. Then, for each positive integer $n$ one has $M\left(S_{\mathfrak{p}^{n}}\right)=M\left(S_{\mathfrak{p}}\right)$.

Proof. We restrict to the case $n=2$, the higher powers behave similarly. Suppose $S_{\mathfrak{p}^{2}}$ to be defined over $M\left(S_{\mathfrak{p}^{2}}\right)$. First,

$$
M\left(S_{\mathfrak{p}^{2}}\right)>M\left(S_{\mathfrak{p}}\right)
$$


because otherwise we would have a $\sigma \in \operatorname{Gal}(\overline{\mathbb{Q}})$ leaving invariant $S_{\mathfrak{p}^{2}}$ but having two non-isomorphic quotient congruence curves

$$
S_{\mathfrak{p}} \quad \text { and } \quad\left(S_{\mathfrak{p}}\right)^{\sigma}
$$

which is impossible.

Second, we get $M\left(S_{\mathfrak{p}^{2}}\right)<M\left(S_{\mathfrak{p}}\right)$ by the following argument. For all $\sigma \in \mathrm{Gal}\left(\overline{\mathbb{Q}} / M\left(S_{\mathfrak{p}}\right)\right)$, the Galois conjugate curve $\left(S_{\mathfrak{p}^{2}}\right)^{\sigma}$ has as many uniform dessins as $S_{\mathfrak{p}^{2}}$, and they are of the same types and have the same automorphism groups as them (up to isomorphism, of course). By the main results of [10], this can occur only if this Galois conjugate curve has a surface group $\Gamma$ contained in a principal congruence subgroup $\Delta\left(\mathfrak{q}^{2}\right)$ with a prime ideal $\mathfrak{q}$ in $k$ of the same norm $q$ as $\mathfrak{p}$. Moreover, since also the index $\left(\Delta: \Delta\left(\mathfrak{p}^{2}\right)\right)$ is invariant under Galois conjugation, we have even $\Gamma=\Delta\left(\mathfrak{q}^{2}\right)$. Now, by the same argument as in the first part of the proof, their common quotient curve $S_{\mathfrak{p}}$ shows

$$
\Delta\left(\mathfrak{q}^{2}\right) \triangleleft \Delta(\mathfrak{p}) \triangleright \Delta\left(\mathfrak{p}^{2}\right)
$$

which is possible only for $\mathfrak{q}=\mathfrak{p}$. Therefore, $\sigma$ sends $S_{\mathfrak{p}^{2}}$ to an isomorphic curve, hence $M\left(S_{\mathfrak{p}^{2}}\right)=M\left(S_{\mathfrak{p}}\right)$.

Lemma 8. Let $\Delta$ be an arithmetically defined cocompact triangle group, containing the norm 1 group of a maximal order of a quaternion algebra $A$ with centre field $k$ (abelian over $\mathbb{Q}$ ), and let $p$ be a rational prime coprime to the discriminant of $A, \mathfrak{p}$ one of the prime ideals of the ring of integers $\mathcal{O}_{k}$ of $k$ in the prime decomposition

$$
p \mathcal{O}_{k}=\left(\mathfrak{p}_{1} \cdot \ldots \cdot \mathfrak{p}_{g}\right)^{e},
$$

$g$ the splitting number and e the ramification index of $p$ in the field extension $k / \mathbb{Q}$. For every positive integer $n$ let $S=S_{\mathfrak{p}^{n}}$ be the quasiplatonic surface with surface group $\Delta\left(\mathfrak{p}^{n}\right)$. Then $[M(S): \mathbb{Q}] \leq g$.

Proof. By Lemma 7 we can restrict ourselves to the case $n=1$. For the action of an arbitrary $\sigma \in \operatorname{Gal}(\overline{\mathbb{Q}})$ on $S$ we know that $S^{\sigma}$ is still quasiplatonic, that regularity, uniformity, and the type of all dessins are preserved. Therefore, if we denote by $\Gamma=\Delta(\mathfrak{p})$ the surface group of $S$ and by $\Gamma_{\sigma}$ the surface group of $S^{\sigma}$, then $\Gamma_{\sigma}$ is again normal in $\Delta$ and $S^{\sigma}$ has one regular dessin and $N(\mathfrak{p})+1$ uniform ones of type $(r, s, t)$ if $\Delta=\Delta(r, s, t)$. By [10] we know that this is only possible if the surface group of $S^{\sigma}$ is contained in one of the principal congruence subgroups $\Delta\left(\mathfrak{p}_{j}\right)$. Because also degrees of Belyi functions are Galois invariant, we have even $\Gamma_{\sigma}=\Delta\left(\mathfrak{p}_{j}\right)$ for some $j$, since

$$
\left(\Delta: \Delta\left(\mathfrak{p}_{j}\right)\right)=(\Delta: \Delta(\mathfrak{p}))=\left(\Delta: \Gamma_{\sigma}\right) \quad .
$$

Therefore the well-known group action of $\operatorname{Gal}(\overline{\mathbb{Q}})$ on the set of prime ideals $\mathfrak{p}_{j}, j=$ $1, \ldots, g$, induces an action on the principal congruence subgroups $\Delta\left(\mathfrak{p}_{j}\right)$ and their corresponding surfaces $S_{\mathfrak{p}_{j}}$ whose stabiliser subgroup $I_{S}$ has an index at most $g$ in $\operatorname{Gal}(\overline{\mathbb{Q}})$. So Galois theory implies the claim about the field degree. 
Corollary 2. Under the same assumptions, we have $[M(S): \mathbb{Q}] \leq[k: \mathbb{Q}]$. Moreover, $M(S)=\mathbb{Q}$ in all cases with splitting number $g=1$, that is in particular for all rational primes $p$ inert or totally ramified in $k$.

This applies in particular to the examples we have already seen: for Bring's curve we have $k=\mathbb{Q}(\sqrt{5})$ with $p=5$ totally ramified, for Klein's quartic $k=\mathbb{Q}\left(2 \cos \frac{2 \pi}{7}\right)$ and $p=7$ is totally ramified too. The next Hurwitz curve (with the same $\Delta=\Delta(2,3,7)$, hence with the same $k$ ) is the Fricke-Macbeath curve $S_{2}$ in genus 7 with surface group $\Delta(2), 2$ inert in $k$, hence with $M\left(S_{2}\right)=\mathbb{Q}$ as well.

Not only in these cases the estimate of Lemma 8 is sharp. Streit's method used in [19] extends to many other arithmetic triangle groups; the technical difficulties in his approach are however not smaller than those in the preprint [2] by Clark and Voight.

\subsection{Fields of definition}

In this section we will study the fields of definition of some of the curves involved in the construction and we will find simultaneous fields of definition for the uniform dessins constructed in the previous sections.

We start by stating some facts about the fields of definition of the intermediate curves $S_{i}$.

Lemma 9. Let $S=S_{\mathfrak{p}}$ be the quasiplatonic surface with surface group $\Delta(\mathfrak{p})$, with $\Delta$ a maximal arithmetic triangle group containing the norm 1 group of a maximal order of $A$ and $\mathfrak{p}$ a prime not dividing the discriminant of $A$, and let $S_{i} \cong \Delta_{0}^{i}(\mathfrak{p}) \backslash \mathbb{H}$ be as above. Then $S_{i}$ can be defined over $M(S)$.

Proof. Let $g_{i j}: S_{i} \rightarrow S_{j}=S_{i}^{\sigma}$ be the unique isomorphism between $S_{i}$ and $S_{j}$ induced by an element $\delta_{i j}$ in the norm 1 group $\Phi$ contained in $\Delta$. This choice is unique because there are precisely $q+1$ residue classes $\delta \Delta_{0}^{i}(\mathfrak{p}) \in \Phi / \Delta_{0}^{i}(\mathfrak{p})$ which give by conjugation the $q+1$ different $\Delta_{0}^{j}(\mathfrak{p})$. Composition of such isomorphisms gives again an isomorphism induced by an element of $\Phi$, and Galois conjugation by $\tau \in \operatorname{Gal}(\overline{\mathbb{Q}} / M(S))$ preserves also this collection of isomorphisms induced by the norm 1 group $\Phi$ because $\Phi / \Delta(\mathfrak{p})$ is the commutator subgroup of the full automorphism group $G:=\Delta / \Delta(\mathfrak{p})$ of $S$. Therefore, the collection of these $g_{i j}$ satisfies Weil's cocycle condition whence all $S_{i}$ can be defined over $M(S)$.

Theorem 2. Under the same hypotheses as in Lemma 9, $M\left(S, \beta_{i}\right)=M(S)$ for all $i$, in other words: the moduli field of $S$ is also a moduli field of all uniform non-regular dessins on it.

Proof. We may suppose $S$ to be defined over $M(S)$. For all $i$ and all $\sigma \in \operatorname{Gal}(\overline{\mathbb{Q}} / M(S))$, $\beta_{i}^{\sigma}$ is another uniform Belyi function $\beta_{j}$ of the same type, and for them the same $\delta_{i j}$ as in Lemma 9 induce automorphisms $d_{i j}:=\delta_{i j} \Delta(\mathfrak{p}) \in G=$ Aut $S$ with the property $\beta_{j} \circ d_{i j}=\beta_{i}$. 
Unfortunately, in contrast to the isomorphisms $g_{i j}$ in the proof of Lemma 9, the automorphisms $d_{i j}$ are uniquely determined only modulo the subgroup $\Delta_{0}^{j}(\mathfrak{p}) / \Delta(\mathfrak{p})$ of $G$, so Weil's criterion does not apply immediately. The question of whether the uniform dessins can be defined over their field of moduli $M(S)$ does not have therefore an obvious answer. However, some partial answers can be given.

Theorem 3. Under the same hypotheses as in Lemma 9, let $S_{i}$ be defined over $M(S)$. Suppose moreover that there exists a $M(S)$-rational point $x \in S_{i}$, not critical for the canonical covering $\tau_{i}: S \rightarrow S_{i}$. Then $\left(S, \beta_{i}\right)$ can be defined over $M(S)$.

Proof. By Lemma 5 we know that $\beta_{i}=r_{i} \circ a_{i} \circ \tau_{i}$. First, let us prove that both $\tau_{i}$ and $r_{i}$ can defined over $M(S)$. Since $S_{i}$ and $x$ are $\operatorname{Gal}(\overline{\mathbb{Q}} / M(S))$-invariant and $\tau_{i}$ is a normal covering, one can apply the method of Coombes and Harbater [4]: take a preimage $y$ of $x$ under $\tau_{i}$. For all $\sigma \in \operatorname{Gal}(\overline{\mathbb{Q}} / M(S))$, we have $\sigma(y)$ in the preimage of $x$ under $\tau_{i}^{\sigma}$, hence there is a unique isomorphism

$$
d_{\sigma}: S \rightarrow S^{\sigma} \quad \text { with } \quad d_{\sigma}(y)=\sigma(y) \quad \text { and } \quad \tau_{i}=\tau_{i}^{\sigma} \circ d_{\sigma}
$$

so Weil's criterion applies to the pair $\left(S, \tau_{i}\right)$. The function $r_{i}$ is also $\operatorname{Gal}(\overline{\mathbb{Q}} / M(S))$ invariant: otherwise we would have several different regular Belyi functions $\beta=r_{i} \circ \tau_{i}$ and $\beta^{\sigma}=r_{i}^{\sigma} \circ \tau_{i}$ on $S$.

Now, the Fricke involution $a_{i}$ of $S_{i}$ is not necessarily fixed by all Galois elements $\sigma \in$ $\operatorname{Gal}(\overline{\mathbb{Q}} / M(S))$. If $a_{i}^{\sigma}=a_{i}$, then $\beta_{i}^{\sigma}=r_{i}^{\sigma} \circ a_{i}^{\sigma} \circ \tau_{i}^{\sigma}=r_{i} \circ a_{i} \circ \tau_{i}=\beta_{i}$.

Let us suppose that $a_{i}^{\sigma} \neq a_{i}$. Note that, if $a_{i}$ is induced by conjugation by $\alpha_{i} \in$ $N\left(\Delta_{0}^{i}(\mathfrak{p})\right)$, then $a_{i}^{\sigma}=a_{i} \circ d$ is induced by conjugation by $\alpha_{i} \delta$ for some $\delta \in N\left(\Delta_{0}^{i}(\mathfrak{p})\right) \cap$ $\alpha_{i} \Delta \alpha_{i}^{-1}$, since $\alpha_{i} \delta$ must interchange the maximal triangle groups $\Delta$ and $\alpha_{i} \Delta \alpha_{i}^{-1}$. But the automorphism group Aut $\left(S_{i}, u_{i}\right)$ of the Belyi function $u_{i}=r_{i} \circ a_{i}$ is induced by the normaliser of $\Delta_{0}^{i}(\mathfrak{p})$ in $\alpha_{i} \Delta \alpha_{i}^{-1}$. As a consequence one has $u_{i} \circ d=u_{i}$ and $\beta_{i}^{\sigma}=r_{i}^{\sigma} \circ a_{i}^{\sigma} \circ \tau_{i}^{\sigma}=$ $r_{i} \circ a_{i} \circ d \circ \tau_{i}=\beta_{i}$.

If we ask for fields over which all relevant functions are defined simultaneously, the results become slightly weaker, of course.

Theorem 4. Under the same hypotheses as in Lemma 9, the regular and all uniform Belyi functions on the Shimura curve $S=S_{\mathfrak{p}}$ can be defined simultaneously over the field of $\operatorname{moduli} M(S, G)$.

Proof. By Corollary 1, the function fields of $S, \mathbb{P}^{1}$ and all $S_{i}$ can be defined over $M(S, G)$, and all quotient maps $\tau_{i}$ and $r_{i}$ in Lemma 5 correspond to the respective embeddings of these function fields into each other. As a consequence all these maps can be defined over $M(S, G)$ simultaneously. Now, by the same argument as in the proof of Theorem 3 , for all $\sigma \in \operatorname{Gal}(\overline{\mathbb{Q}} / M(S, G))$ one has $u_{i}^{\sigma}=u_{i}$, hence also $\beta_{i}^{\sigma}=\beta_{i}$.

Example 3. Let $\mathcal{B} \cong \mathbb{H} / \Delta(\sqrt{5})$ be Bring's curve already introduced in Example 2. Let us recall that its full automorphism group Aut $\mathcal{B} \cong \mathfrak{S}_{5}$ acts on the point $\left[x_{0}, \ldots, x_{4}\right] \in \mathcal{B}$ by 
permutation of the projective coordinates. Since both $\mathcal{B}$ and its automorphism group are defined over $\mathbb{Q}$, by Theorem 4 its regular dessin and all its uniform dessins can be defined over $\mathbb{Q}$ (even simultaneously).

Example 4. In the affine model $\mathcal{K}_{F}:=\left\{y^{7}=x(x-1)^{2}\right\}$ of Klein's quartic $\mathcal{K}$ (see Example $2)$, the function

$$
\beta_{0}(x, y)=-\frac{\left(x^{6}-235 x^{5}+1430 x^{4}-1695 x^{3}+270 x^{2}+229 x+1\right)^{3}\left(x^{2}-x+1\right)^{3}}{1728 x(x-1)\left(x^{3}+5 x^{2}-8 x+1\right)^{7}}
$$

is one of the $(2,3,7)$ uniform Belyi functions (see Example 4.44 in [9]) and it is defined over $M(\mathcal{K})=\mathbb{Q}$. However, in this model there are automorphisms not defined over $\mathbb{Q}$, and therefore we cannot expect all uniform Belyi functions in $\mathcal{K}_{F}$ to be defined over $\mathbb{Q}$ simultaneously.

However, there is a model for Klein's quartic in which all uniform Belyi functions are simultaneously defined over the field $\mathbb{Q}(\sqrt{-7})$. This follows from a result in Clark and Voight's preprint [2] saying that in this case $M(\mathcal{K}, G)=\mathbb{Q}(\sqrt{-7})$. A relatively easy proof for this claim is the following.

Another model for $\mathcal{K}$ defined over the rationals is the projective equation $x^{3} y+y^{3} z+$ $z^{3} x=0$, and in this model all automorphisms are defined over the cyclotomic field $\mathbb{Q}\left(\zeta_{7}\right)$, therefore $M(\mathcal{K}, G)$ has to be a subfield of this cyclotomic field. The absolute Galois group acts as automorphism group on $G \cong \operatorname{PSL}\left(2, \mathbb{F}_{7}\right)$ whose automorphism group is isomorphic to $\operatorname{PGL}\left(2, \mathbb{F}_{7}\right)$, an index 2 extension of its inner automorphism group $\cong G$. For inner automorphisms, that is for those $\sigma$ acting on $G$ as

$$
\alpha \mapsto \alpha^{\sigma}=\gamma^{-1} \alpha \gamma \quad \text { for some } \quad \gamma=f_{\sigma} \in G
$$

we have obviously $\sigma \in I_{(\mathcal{K}, G)}$, therefore $M(\mathcal{K}, G)$ can be an extension of $\mathbb{Q}$ of degree at most 2 . Because $\mathbb{Q}(\sqrt{-7})$ is the only quadratic subfield of $\mathbb{Q}\left(\zeta_{7}\right)$, the claim follows.

In a similar way - using Lemma 6 instead of Lemma 5 - we can prove

Theorem 5. For all prime ideal powers coprime to the discriminant of $A$, the regular and all uniform Belyi functions on the Shimura curve $S=S_{\mathfrak{p}^{n}}$ can be defined simultaneously over the field of moduli $M(S, G)$.

\section{Appendix: the role of the splitting field}

Theorem 6. Under the assumptions of Lemma 8, but with the possible exception of the case $\Delta=\Delta(3,4,6), M(S)$ is the splitting field of $p$ in $k$, that is the fixed field of all $\sigma \in$ Gal $k / \mathbb{Q}$ fixing the prime ideals $\mathfrak{p}_{j} \mid p$. The action of the absolute Galois group on these principal congruence subgroup Shimura curves is the same as the action on the level ideals: if the principal congruence subgroup $\Delta\left(\mathfrak{p}^{n}\right)$ is the surface group of $S:=S_{\mathfrak{p}^{n}}, \Delta\left(\sigma\left(\mathfrak{p}^{n}\right)\right)$ is the surface group $\Delta\left(\mathfrak{p}^{n}\right)_{\sigma}$ of $S^{\sigma}$. The field of moduli $M(S)$ coincides with the field of moduli $M(\mathcal{D})$ of the maximal regular dessin on $S$. 
This splitting field can also be considered as the smallest subfield of $k$ (of degree $g$ ) in which $p$ splits in $g$ prime ideals (in this splitting field necessarily of residue class degree 1 ).

Proof. 1. With Lemma 7, the result clearly extends from $\mathfrak{p}$ to all prime power levels $\mathfrak{p}^{n}$, so we will consider only the case $n=1$. Another simplification comes from Lemma 1: since $\Delta$ is maximal, we have in fact $M(\mathcal{D})=M(S)$. So it is sufficient to care about $M(S)$.

2. As a preparatory step, we need to learn more about the full automorphism group $G:=\Delta / \Delta(\mathfrak{p})$ of $S:=S_{\mathfrak{p}}$. All triangle groups in question are either the norm 1 groups $\Phi$ mentioned in Lemma 8 or extensions of them of degree 2 or 4 . In a few cases, $\Phi$ is not a triangle group itself but a quadrangle group. The extensions are generated by integer elements $\delta \in A$ of totally positive norm $\nu \in k$, either a prime dividing the discriminant $D(A)$ or a non-square unit of $k$. The arithmetic in $A$ implies that $\delta$ normalizes congruence subgroups $\Delta(\mathfrak{p})$ as well. If $q$ denotes the norm $N(\mathfrak{p})$ and if $\Delta$ is generated by $\Phi$ and $\delta$, then $\Phi / \Delta(\mathfrak{p}) \cong \operatorname{PSL}\left(2, \mathbb{F}_{q}\right)$ and $\Delta / \Delta(\mathfrak{p}) \cong \operatorname{PSL}\left(2, \mathbb{F}_{q}\right) \times C_{2}$ or $\cong \operatorname{PGL}\left(2, \mathbb{F}_{q}\right)$ depending on the alternative whether the (reduced quaternion) norm $N(\delta) \bmod \mathfrak{p}$ is a square in $\mathbb{F}_{q}$ or not. If $\Delta$ is an index 4 extension of $\Phi$, the quotient $\Delta / \Delta(\mathfrak{p})$ is $\cong \operatorname{PSL}\left(2, \mathbb{F}_{q}\right) \times C_{2} \times C_{2}$ or $\cong \operatorname{PGL}\left(2, \mathbb{F}_{q}\right) \times C_{2}$ by a similar argument (we learned this idea from A. Džambić).

3. By the arguments already used in the proof of Lemma 8, we know that the quasiplatonic surfaces $S_{\mathfrak{p}_{j}}, j=1, \ldots, g$, form a family $\mathcal{F}$ invariant under the action of the absolute Galois group $\operatorname{Gal}(\overline{\mathbb{Q}})$, so the splitting number $g$ is an upper bound for the length of the Galois orbit of $S$. We can suppose that all these surfaces are equipped with the dessins induced by the maximal triangle group $\Delta \triangleright \Phi \triangleright \Delta\left(\mathfrak{p}_{j}\right)$ of signature $(r, s, t)$. Let $\mathfrak{p}$ be one of these prime ideals. Let $G$ be the automorphism group of $S:=S_{\mathfrak{p}}$, generated by the elements $g_{0}, g_{1}, g_{\infty}$ of respective orders $r, s, t$, images of the canonical generators $\gamma_{0}, \gamma_{1}, \gamma_{\infty}$ of $\Delta$ under the canonical epimorphism

$$
h: \Delta \rightarrow \Delta / \Delta(\mathfrak{p}) \cong G .
$$

If $g_{1}$, say, has a fixed point $P \in S$ and acts in suitable local coordinates on a neighbourhood of $P$ like

$$
g_{1}: z \mapsto \zeta_{s}^{v} \cdot z,
$$

we call $\zeta_{s}^{v}$ the multiplier of $g_{1}$ in $P$. Clearly, $v$ is coprime to $s$. The collections of all pairs

$$
\left(\zeta_{r}^{u}, n_{r, u}\right), \quad\left(\zeta_{s}^{v}, n_{s, v}\right), \quad\left(\zeta_{t}^{w}, n_{t, w}\right)
$$

are called the multiplier data of $\left(G, g_{0}, g_{1}, g_{\infty}\right)$ on $S$ where we denote by $n_{r, u}$ the number of all fixpoints of $g_{0}$ on $S$ with multiplier $\zeta_{r}^{u}$, and so on.

4. As next step we show how the action of the absolute Galois group on the family $\mathcal{F}$ induces an action on the multiplier data. For all $\sigma \in \operatorname{Gal}(\overline{\mathbb{Q}})$ the multiplier data of $\left(G^{\sigma}, g_{0}^{\sigma}, g_{1}^{\sigma}, g_{\infty}^{\sigma}\right)$ arise from the original ones by an obvious action of $\sigma$ on the multipliers, see [19] or [13]. We can simplify the consideration of this Galois action on the multiplier data in two ways. First, we can neglect all multipliers with (say) $r=2$ because $\operatorname{Gal}(\overline{\mathbb{Q}})$ acts trivially on $\zeta_{2}=-1$. Second, we can exclude all primes $p$ from the consideration in our theorem which divide one of the entries of the signature of $\Delta$ : a case-by-case analysis 
of all 19 triangle groups in question (see Table (3) of [20]) shows that this possibility occurs only if $p$ and the discriminant ideal $D(A)$ of the algebra have a nontrivial common divisor or - much more often - if the splitting number of $p$ in $k$ is $g=1$, so Corollary 2 applies. Instead of a tedious list we give two typical examples.

a) $\Delta=\Delta(2,5,6)$ with $k=\mathbb{Q}(\sqrt{5})$. Here, 2 divides the discriminant of the algebra, 3 is inert and 5 is ramified.

b) $\Delta=\Delta(2,5,30)$ with $k=\mathbb{Q}\left(\cos \frac{\pi}{15}\right)$, the maximal real subfield of $\mathbb{Q}\left(\zeta_{15}\right)$, is a bit more complicated. The prime 3 divides the discriminant $D(A)$. The Galois group of $\mathbb{Q}\left(\zeta_{15}\right) / \mathbb{Q}$ is isomorphic to $(\mathbb{Z} / 15 \mathbb{Z})^{*} \cong\{ \pm 1 \bmod 15\} \times\{1,2,4,8 \bmod 15\}$, and $k$ is the fixed field of the first factor, and therefore

$$
\operatorname{Gal}(k / \mathbb{Q}) \cong(\mathbb{Z} / 15 \mathbb{Z})^{*} /\{ \pm 1\} \cong\{1,2,4,8 \bmod 15\}
$$

This second factor is generated by the Frobenius automorphism for the prime 2 , so this prime is inert in $k$. Finally, the prime 5 is totally ramified already in the subfield $\mathbb{Q}\left(\zeta_{5}\right)$ and inert in the subfield $\mathbb{Q}\left(\zeta_{3}\right)$, hence it has in $\mathbb{Q}\left(\zeta_{15}\right)$ the decomposition $5 \mathbb{Z}\left[\zeta_{15}\right]=\mathfrak{P}^{4}$ with one prime ideal $\mathfrak{P}<\mathbb{Z}\left[\zeta_{15}\right]$ of residue degree 2 . Because the splitting number of 5 is $g=1$ in $\mathbb{Q}\left(\zeta_{15}\right)$, it is also 1 in its subfield $k$.

The primes not dividing the signature entries have the advantage that they cannot belong to parabolic generators of $\mathrm{SL}\left(2, \mathbb{F}_{q}\right)$ or $\mathrm{GL}\left(2, \mathbb{F}_{q}\right)$; these are the only ones having eigenvalues of multiplicity 2 . So, for the other primes $p$ our generators $g_{i}$ (that is $g_{0}, g_{1}$ or $\left.g_{\infty}\right)$ are non-parabolic, therefore $g_{i}$ is conjugate in $G$ to $g_{i}^{ \pm 1}$ but to no other power of $g_{i}-$ an easy consequence of the structure of $\operatorname{SL}\left(2, \mathbb{F}_{q}\right)$ and $\operatorname{GL}\left(2, \mathbb{F}_{q}\right)$, compare the eigenvalues of their matrices and the respective arguments already used in [19] and [8]. By construction of (say) $g_{i}=g_{\infty}=h\left(\gamma_{\infty}\right)$ by means of the canonical epimorphism $h$, the fixpoint $u \in \mathbb{H}$ of $\gamma_{\infty}$ gives at least one fixpoint $P=\Delta(\mathfrak{p}) u \in S=\Delta(\mathfrak{p}) \backslash \mathbb{H}$ with multiplier $\zeta_{t}$. Now suppose that $g_{\infty}$ fixes another point $Q \in S$, then this is a fixed point of the same order because $\Delta$ is maximal and so the orders of the generators of $G$ (the signature entries) are pairwise different. So, $Q$ and $P$ are both face centers of the dessin (or vertices of the same colour in the cases $i=0,1)$. By the transitivity of $G$ there is an automorphism $a \in G$ with $a(P)=Q$, therefore $a^{-1} g_{\infty} a$ fixes $P$ as well, hence is conjugate to a power of $g_{\infty}$, and we know that here $g_{\infty}^{ \pm 1}$ are the only possible powers. Since the multiplier of $g_{\infty}$ in $Q$ is the same as that of $a^{-1} g_{\infty} a$ in $P$, it has to be $\zeta_{t}^{ \pm 1}$. The only possible contribution of $g_{\infty}$ to the multiplier data are therefore the pairs

$$
\left(\zeta_{t}, n_{t, 1}\right) \quad \text { and } \quad\left(\zeta_{t}^{-1}, n_{t,-1}\right)
$$

In the following, we will therefore always assume (without loss of generality, see above) that $p$ is coprime to $r, s, t$. As a consequence, $p$ is unramified in all cyclotomic fields in question, hence also unramified in $k$, see the next step of the proof.

5. By the same proof as in [19] we can moreover see that $n_{t, 1}=n_{t,-1}$ : consider the canonical representation $\psi$ of $G$ on the space of holomorphic differentials of $S$. Since $g_{\infty}$ is conjugate to $g_{\infty}^{-1}$, and since

$$
\operatorname{tr} \psi\left(g_{\infty}\right)=\operatorname{tr} \psi\left(g_{\infty}^{-1}\right)=\operatorname{tr} \psi\left(g_{\infty}\right)^{-1}=\overline{\operatorname{tr} \psi\left(g_{\infty}\right)}
$$


$\psi\left(g_{\infty}\right)$ has a real trace. On the other hand, Eichler's trace formula

$$
\operatorname{tr} \psi\left(g_{\infty}\right)=1+n_{t, 1} \frac{\zeta_{t}}{1-\zeta_{t}}+n_{t,-1} \frac{\zeta_{t}^{-1}}{1-\zeta_{t}^{-1}}
$$

gives a real value if and only if $n_{t, 1}=n_{t,-1}$, so the multiplier system is invariant under complex conjugation. Obviously, the action of $\operatorname{Gal}(\overline{\mathbb{Q}})$ on the multiplier data corresponds therefore to the action on $\mathbb{Q}\left(\cos \frac{2 \pi}{r}, \cos \frac{2 \pi}{s}, \cos \frac{2 \pi}{t}\right)$, and this is precisely the center field $k$ of the quaternion algebra $A$ for all maximal arithmetic triangle groups except $\Delta(3,4,6)$. This can again be seen via a case-by-case analysis along the lines of Takeuchi's Table (3) in [20]. Two consequences are important: first, $k<\mathbb{Q}\left(\zeta_{r}, \zeta_{s}, \zeta_{t}\right)$, therefore (by the assumptions justified in step 4 of the proof) $p$ is also unramified in $k$; in other words, the exponent $e=1$ in the prime decomposition of Lemma 8. Second, on $S^{\sigma}$ the contribution of the Galois conjugate generator $g_{\infty}^{\sigma}$ with $\sigma\left(\zeta_{t}\right)=\zeta_{t}^{w}$ to the multiplier data is $\left(\zeta_{t}^{w}, n_{t, 1}\right),\left(\zeta_{t}^{-w}, n_{t, 1}\right)$. Together with the analogous facts for the other generators and with $\zeta_{t}+\zeta_{t}^{-1}=2 \cos \left(\frac{2 \pi}{t}\right)$ the action of the absolute Galois group $\operatorname{Gal}(\overline{\mathbb{Q}})$ on the multiplier data gives an orbit of length $[k: \mathbb{Q}]$.

6. In general, this orbit length is however not the orbit length of the action of $\operatorname{Gal}(\overline{\mathbb{Q}})$ on the family $\mathcal{F}$ of the Shimura congruence curves $S_{\mathfrak{p}_{j}}, j=1, \ldots, g$ : if we consider again the canonical homomorphism $h: \Delta \rightarrow G=\Delta / \Delta(\mathfrak{p})=$ Aut $S$, we get in fact at least $[k: \mathbb{Q}]$ different epimorphisms $\sigma \circ h$, but their kernels coincide if and only if they differ by composition with an automorphism of $G$. (Here we use again the hypothesis that $\Delta$ is maximal, so we cannot pass to other homomorphisms by permutation of the generators.) Since we need only a lower bound for the length of the Galois orbit (remember step 3 above), it is sufficient to study these automorphisms on its commutator subgroup $[G, G]=\operatorname{PSL}\left(2, \mathbb{F}_{q}\right)$ or its extension $\operatorname{PGL}\left(2, \mathbb{F}_{q}\right)$ - the generators of the possible $C_{2}$ factors are anyway irrelevant for the Galois action, see step 4 . The automorphisms of these matrix groups over $\mathbb{F}_{q}$ are composed by

- matrix conjugations leaving eigenvalues and traces invariant - so they leave invariant the multipliers - and

- Galois conjugations by $\operatorname{Gal}\left(\mathbb{F}_{q} / \mathbb{F}_{p}\right)$ acting on the matrix coefficients and hence also on the eigenvalues.

If $\sigma \in \operatorname{Gal}(\overline{\mathbb{Q}})$ induces this second kind of automorphism of $G$, it sends therefore a generator $g_{i} \in G, i=0,1, \infty$, to another element conjugate to some power $g_{i}^{ \pm w}$ where $w$ is some $p$-power (here and in the following neglecting possible $C_{2}$ factors, see above).

7. This is true in the same way for the $g_{i}$ as matrices over $\mathbb{F}_{q}$ and for the $g_{i}$ as automorphisms of $S$. In the fist version, $\sigma$ induces an action on their eigenvalues in the finite fields $\mathbb{F}_{p^{m}}$, in the second version an analogous action

$$
\zeta_{i}+\zeta_{i}^{-1} \mapsto \zeta_{i}^{w}+\zeta_{i}^{-w}, \quad w \quad \text { a } p \text {-power }
$$


where $\zeta_{i}$ denotes the multiplier of $g_{i}$. Clearly, if $\sigma$ fixes $k$ elementwise, it has this behaviour (with $w= \pm 1$ ). Recall from number theory in cyclotomic fields that the Frobenius subgroup of $\operatorname{Gal}\left(\mathbb{Q}\left(\zeta_{i}\right) / \mathbb{Q}\right)$ consisting of all

$$
\sigma: \zeta_{i} \mapsto \zeta_{i}^{w}, \quad w \text { a } p \text {-power, }
$$

is precisely the maximal subgroup fixing the prime ideals in the prime decomposition of the (unramified!) rational prime $p$. Its fixed field is the splitting field of $p$ in $\mathbb{Q}\left(\zeta_{i}\right)$. Using this fact for all three cyclotomic fields $\mathbb{Q}\left(\zeta_{i}\right)$, the restriction to $k$ is an exercise in Galois theory and shows

$$
\left\{\sigma \in \operatorname{Gal}(\overline{\mathbb{Q}}) \mid S \cong S^{\sigma}\right\} \leq U_{p}:=\{\sigma \in \operatorname{Gal}(\overline{\mathbb{Q}}) \mid \sigma(\mathfrak{p})=\mathfrak{p}\}
$$

( $U_{p}$ depending only on $k$ and $p$, not on the choice of $\mathfrak{p}$ among the $\mathfrak{p}_{j}$ because $k$ is abelian). The orbit of $\operatorname{Gal}(\overline{\mathbb{Q}})$ on the family $\mathcal{F}$ has therefore at least length $\left|\operatorname{Gal}(\overline{\mathbb{Q}}): U_{p}\right|$, and since the splitting field $K_{p} \leq k$ of $p$ is the fixed field of $U_{p}$, this group index is the field degree $\left[K_{p}: \mathbb{Q}\right]=g$. Together with the upper bound for the Galois orbit given in Lemma 8 we see therefore that $K_{p}$ is in fact the field of moduli - hence also the minimal field of definition - of $S$.

8. As a side result, we see also that the multiplier data determine uniquely the curves of the family $\mathcal{F}$. Therefore, Proposition 3 of [13] shows that the actions of $\operatorname{Gal}(\overline{\mathbb{Q}})$ on $\mathcal{F}$, their multiplier data, and their corresponding prime ideals $\mathfrak{p}_{j}$ are compatible.

Remark 3. Step 5 of the proof above fails for $\Delta=\Delta(3,4,6)$ because the centre field $k=$ $\mathbb{Q}(\sqrt{6}) \neq \mathbb{Q}\left(\cos \frac{2 \pi}{r}, \cos \frac{2 \pi}{s}, \cos \frac{2 \pi}{t}\right)=\mathbb{Q}$. Moreover, the minimal cyclotomic field containing $k$ is generated by the 24-th root of unity $\zeta_{24}$, hence has degree 4 over $k$. Therefore, no group commensurable with $\Delta$ can contain a torsion element $\gamma$ of order $m \neq 2,3,4,6$ because otherwise we would have a cyclotomic subfield $k(\gamma) \cong k\left(\zeta_{m}\right)<A$, and this can have at most degree 2 over $k$ because A cannot contain larger commutative subfields.

Is it possible that in this case all $S_{\mathfrak{p}}$ are defined over $\mathbb{Q}$ ? No: by the quadratic reciprocity law, rational primes $p$ split in $k$ if and only if $p \equiv \pm 1$ or $\pm 5 \bmod 24$. The first example $p=5, \mathfrak{p}=(1 \pm \sqrt{6}) \mathcal{O}_{k}$ gives two Galois conjugate curves of genus 16 with field of moduli $k$, see the (quite different) proof in [3].

\section{References}

[1] Belyı̆, G.V., On Galois extensions of a maximal cyclotomic field, Izv. Akad. Nauk SSSR Ser. Mat. 43 (1979), 267-276, 479.

[2] Clark, P., Voight, J., Algebraic curves uniformized by congruence subgroups of triangle groups, preprint, available at the authors' web pages. 
[3] Conder, M.D.E., Jones, G.A., Streit, M., Wolfart, J.: Galois actions on regular dessins of small genera, Revista Mat. Iberoamericana 29 (2013), 163-181.

[4] Coombes, K., Harbater, D., Hurwitz families and arithmetic Galois groups, Duke Math. J. 52 (1985) 821-839.

[5] Dèbes, P., Douai, J.-C., Algebraic covers: field of moduli versus field of definition, Ann. scient. Ec. Norm. Sup. 4e série, 30 (1997), 303-338.

[6] Džambić, A., Macbeath's infinite series of Hurwitz groups. In: Arithmetic and geometry around hypergeometric functions, ed. Holzapfel, R.-P., Uludag, A.M., Yoshida, M. (Progr. Math., 260, Birkhäuser, Basel, 2007), pp. 101-108.

[7] Earle, C.J., On the Moduli of Closed Riemann Surfaces with Symmetries. In: Advances in the Theory of Riemann Surfaces, ed. L.V. Ahlfors et al. (Ann. of Math. Studies 66, Princeton, 1971), pp. 119-130.

[8] Feierabend, F.: Galois-Operationen auf verallgemeinerten Macbeath-Hurwitz Kurven, PhD thesis, Frankfurt 2008.

[9] Girondo, E., González-Diez, G., Introduction to compact Riemann surfaces and dessins d'enfants, London Mathematical Society Student Texts 79, Cambridge University Press, Cambridge, 2011.

[10] Girondo, E., Torres-Teigell, D., Wolfart, J., Shimura curves with many uniform dessins, Math. Z. 271 (2012), n. 3-4, 757-779.

[11] Girondo, E., Wolfart, J., Conjugators of Fuchsian groups and quasiplatonic surfaces, Quarterly J. Math. 56 (2005), 525-540.

[12] Grothendieck, A., Esquisse d'un Programme. In: Geometric Galois Actions. 1. Around Grothendieck's Esquisse d'un Programme, ed. L. Schneps and P. Lochak (London Math. Soc. Lecture Note Ser. 242, Cambridge University Press, 1997), pp. 5-48.

[13] Jones,G.A., Streit, M., Wolfart, J., Wilson's map operations on regular dessins and cyclotomic fields of definition, Proc. London Math. Soc. 100 (2010), 510-532.

[14] Maclachlan, C., Reid, A. W., The arithmetic of hyperbolic 3-manifolds. Graduate Texts in Mathematics, 219. Springer-Verlag, New York, 2003.

[15] Macbeath, A.M., Generators of the Fractional Linear Groups. In: 1969 Number Theory (Proc. Sympos. Pure Math., Vol. XII, Houston, Tex., 1967), pp. 14-32, Amer. Math. Soc., Providence, R.I.

[16] Riera, G., Rodríguez, R. E., The period matrix of Bring's curve, Pacific J. Math. 154 (1992), no. 1, 179-200. 
[17] Shimura, G., On the field of rationality of an abelian variety, Nagoya Math. J. 45 (1972), 167-178.

[18] Singerman, D., Automorphisms of maps, permutation groups and Riemann surfaces, Bull. London Math. Soc. 8 (1976), 65-68.

[19] Streit, M., Field of definition and Galois orbits for the Macbeath-Hurwitz curves, Arch. Math. 74 (2000) 342-349.

[20] Takeuchi, K., Commensurability classes of arithmetic triangle groups, J. Fac. Sci. Univ. Tokio, Sect. 1A Math. (1) 24 (1977), 201-212.

[21] Vignéras, M.-F., Arithmétique des Algèbres de Quaternions. LNM 800, Springer (1980).

[22] Weil, A., The field of definition of a variety, Amer. J. Math. 78 (1956), 509-524.

[23] Wolfart, J., ABC for polynomials, dessins d'enfants and uniformization - a survey. Elementare und analytische Zahlentheorie, Schr. Wiss. Ges. Johann Wolfgang Goethe Univ. Frankfurt am Main, 20, Franz Steiner Verlag Stuttgart, Stuttgart, 2006, pp. 313-345, also available at http://www. math.uni-frankfurt.de/ wolfart/ 